\title{
Conditioning Clinical Trial Setting
}

National Cancer Institute

\section{Source}

National Cancer Institute. Conditioning Clinical Trial Setting. NCI Thesaurus. Code C156597.

A clinical trial or treatment setting in which the therapy of interest is considered conditioning therapy. 